\title{
Formation of disinfection by-products during sodium hypochlorite cleaning of fouled membranes from membrane bioreactors
}

\author{
Hao Wang ${ }^{1}$, Defang Ma $\left.(\bowtie)\right)^{1}$, Weiye Shi ${ }^{2,3}$, Zhiyu Yang ${ }^{1}$, Yun Cai ${ }^{1}$, Baoyu Gao ${ }^{1}$ \\ 1 Shandong Key Laboratory of Water Pollution Control and Resource Reuse, School of Environmental Science and Engineering, \\ Shandong University, Qingdao 266237, China \\ 2 No.1 Institute of Geology and Mineral Resources of Shandong Province, Jinan 250014, China \\ 3 Shandong Engineering Laboratory for High-Grade Fe Ores Exploration and Exploitation, Jinan 250014, China
}

\section{H I G H L I G H T S}

- HAAs was dominant among the DBPs of interest.

- Rising time, dose, temperature and $\mathrm{pH}$ raised TCM and HAAs but reduced HANs and HKs.

- Low time, dose and temperature and nonneutrality $\mathrm{pH}$ reduced toxic risks of DBPs.

- The presence of EPS decelerated the production of DBPs.

-EPS, particularly polysaccharides were highly resistant to chlorine.

\section{A R T I C L E I N F O}

\section{Article history:}

Received 15 July 2020

Revised 15 October 2020

Accepted 11 November 2020

Available online 10 January 2021

\section{Keywords:}

MBR

Biofouling

EPS

Chemical cleaning

DBPs

CLSM

\section{GR A PHIC A B S T R A T}

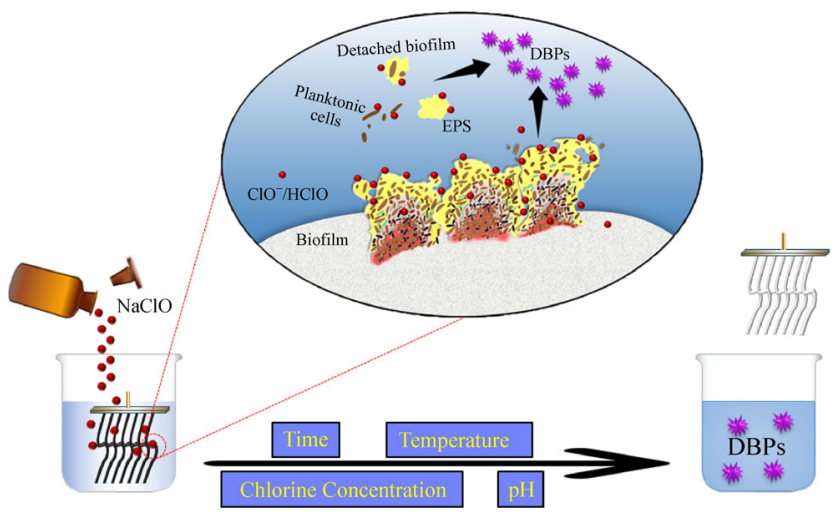

A B S T R A C T

Periodic chemical cleaning with sodium hypochlorite $(\mathrm{NaClO})$ is essential to restore the membrane permeability in a membrane bioreactor (MBR). However, the chlorination of membrane foulants results in the formation of disinfection by-products (DBPs), which will cause the deterioration of the MBR effluent and increase the antibiotic resistance in bacteria in the MBR tank. In this study, the formation of 14 DBPs during chemical cleaning of fouled MBR membrane modules was investigated. Together with the effects of biofilm extracellular polymeric substances (EPS), influences of reaction time, $\mathrm{NaClO}$ dosage, initial $\mathrm{pH}$, and cleaning temperature on the DBP formation were investigated. Haloacetic acids (HAAs) and trichloromethane (TCM), composed over 90\% of the DBPs, were increasingly accumulated as the $\mathrm{NaClO}$ cleaning time extended. By increasing the chlorine dosage, temperature, and $\mathrm{pH}$, the yield of TCM and dichloroacetic acid (DCAA) was increased by up to a factor of 1-14, whereas the yields of haloacetonitriles (HANs) and haloketones (HKs) were decreased. Either decreasing in the chlorine dosage and cleaning temperature or adjusting the $\mathrm{pH}$ of cleaning reagents toward acidic or alkaline could effectively reduce the toxic risks caused by DBPs. After the EPS extraction pretreatment, the formation of DBPs was accelerated in the first $12 \mathrm{~h}$ due to the damage of biofilm structure. Confocal laser scanning microscopy (CLSM) images showed that EPS, particularly polysaccharides, were highly resistant to chlorine and might be able to protect the cells exposed to chlorination.

\section{Introduction}

The membrane bioreactor (MBR) has gained extensive

$凶$ Corresponding author

E-mail: defangma@sdu.edu.cn attention due to the growing needs for water reuse. However, inevitable membrane fouling is still the primary obstacle for its wide application. In MBR, membrane fouling is mainly ascribed to the microorganisms and their extracellular polymeric substances (EPS), which results in the formation of biofilm on the membrane surface (Xiao 
et al., 2014). Although a lot of efforts have been made to relieve MBR membrane fouling (Guo et al., 2019; Ding et al., 2020a; Ding et al., 2020b), membrane cleaning is essential to maintain a stable operation of MBR. Regular online maintenance cleaning and offline recovery cleaning with sodium hypochlorite $(\mathrm{NaClO})$ has been widely adopted in practice because $\mathrm{NaClO}$ has high efficiency for sterilization as well as oxidiation of organic matter, which can effectively remove the biofilm on the surface of the membrane and restore the membrane flux.

Online maintenance cleaning is carried out by backflushing with $\mathrm{NaClO}$ solution at the concentration of several dozen $\mathrm{mg} / \mathrm{L}$ to several hundred $\mathrm{mg} / \mathrm{L}$ for hours once a few days to achieve a stable constant flux. Therefore, it is inevitably that activated sludge in MBR directly contact with residual $\mathrm{NaClO}$ diffused across membranes. Recent studies showed that residual $\mathrm{NaClO}$ could react with the activated sludge mixture during online maintenance cleaning to produce large amounts of disinfection byproducts (DBPs), such as trihalomethanes (THMs), haloacetic acid (HAAs), haloacetonitriles (HANs) and haloketones (HKs), which have genotoxicity, cytotoxicity and carcinogenicity (Wu et al., 2020). DBPs are generally refractory compounds with low molecular weight (MW), which are difficult to be biodegraded by activated sludge or hardly rejected by ultrafiltration/microfiltration membranes in MBR. It was reported that $62.4 \%-84.5 \%$ DBPs generated during online $\mathrm{NaClO}$ cleaning was found in MBR effluents (Cai et al., 2017), which will result in the deterioration of receiving water bodies and eventually affect the downstream drinking water quality (Zhang and Liu, 2020). To control the formation of halogenated DBPs, chlorine-free oxidants, like $\mathrm{Fe}^{2+}$ activated PMS and ozone, were considered as alternative chemical cleaning agents for MBRs (Sun et al., 2018; Wang et al., 2020b).

After finite cycles of operation (usually 1 month to 3 years), the membrane flux cannot be recovered through online maintenance cleaning. Therefore, offline recovery cleaning is generally required to remove the residual fouling that is resistant to online maintenance cleaning to restore the membrane permeability. During offline recovery cleaning, the fouled membranes are immersed in the $\mathrm{NaClO}$ solution with a concentration higher than several thousand milligrams per liter for 8 to $24 \mathrm{~h}$. High concentrations of $\mathrm{NaClO}$ will react with the biofilm on the membrane surface to form DBPs, producing a large quantity of hazardous cleaning agents containing toxic DBPs. In most MBR plants in China, the waste cleaning agents containing high concentrations of DBPs is mixed with the influent wastewater and flow into the MBR reactor, resulting in the direct contact of DBPs and bacteria. Exposure to DBPs may induce or increase antibiotic resistance in bacteria (Lv et al., 2014). Above all, studying the formation of the DBPs during membrane chemical cleaning is necessary for probing the composition of toxicants in MBR permeate and understanding the risks in MBR technologies at present.

The generation and fate of DBPs during the online cleaning process, deriving from the contact reaction of sodium hypochlorite and activated sludge mixture in the MBR reactor have been revealed (Cai et al., 2017; Cai et al., 2020). However, the formation of DBPs derived from the chlorination of biofilm on the MBR membrane surface are still unclear. Note that, the reaction of $\mathrm{NaClO}$ and biofilm on the membrane surface is the necessary step for membrane flux recovery during membrane cleaning. DBPs generated from the chlorination of biofilm on the membrane surface are inevitable in MBR, not only in the online cleaning process but also off-line cleaning process. Microbial species and their EPS in the biofilm on membrane surface are different from those in suspended activated sludge (Garrido-Baserba et al., 2016), which affect the susceptibility to $\mathrm{NaClO}$ (Berry et al., 2010) and will impact the generation of DBPs during disinfection.

Therefore, this study aimed to investigate the formation of DBPs during $\mathrm{NaClO}$ chemical cleaning of fouled membrane modules in a bench-scale MBR. For this purpose, factors affecting the formation of DBPs during the treatment of membrane fouling with $\mathrm{NaClO}$, such as reaction time, chlorine dosage, initial $\mathrm{pH}$ and temperature were comprehensively evaluated. The contribution of EPS in biofilm to the formation of DBPs was also investigated because $80 \%-90 \%$ of the total organic matter in a biofilm is composed of EPS like polysaccharides, proteins, lipids, and nucleic acids, which have been confirmed as precursors of DBPs. The changes of components and structure of biofilm on the membrane surface and the membrane permeability recovery were also determined during the $\mathrm{NaClO}$ cleaning process, to balance the membrane permeability recovery and formation of DBPs in real-life MBR.

\section{Materials and methods}

\subsection{Membrane bioreactors}

Six identical laboratory-scale submerged MBRs were operated to treat simulated domestic sewage. The characteristics of simulated domestic sewage are shown in Supporting Information Table SI-1. The reactor with an effective volume of $12 \mathrm{~L}$ was equipped with a $\mathrm{PVP} / \mathrm{SiO}_{2}$ modified hollow fiber PVDF membrane module (normalized pore size, $0.02 \mu \mathrm{m}$; effective membrane area, $0.075 \mathrm{~m}^{2}$ ), which was purchased from Xiamen Kymem Technology Co., Ltd. The membrane flux was $20 \mathrm{~L} /\left(\mathrm{m}^{2} \mathrm{~h}\right)$. The MBR was operated at ambient temperature $\left(25 \pm 2^{\circ} \mathrm{C}\right)$ with the hydraulic retention time (HRT) of $8 \mathrm{~h}$ and sludge retention time (SRT) of 30 days. The dissolved oxygen in the mixture was maintained at $3.0 \pm 1.0 \mathrm{mg} / \mathrm{L}$. The membrane module was periodically backwashed with effluent to ensure that the trans-membrane pressure (TMP) 
was below $50 \mathrm{kPa}$. Once the TMP could not be maintained below $50 \mathrm{kPa}$ by backflushing, the membrane module was taken out for chemical cleaning treatment.

For the convenience of showing the detachment process of biofouling layer by using confocal laser scanning microscopy (CLSM) and investigating the membrane flux recovery during $\mathrm{NaClO}$ cleaning, a crossflow plate MBR was applied to grow biofilm on the membrane surface. Detailed information is shown in Supporting Information Text SI-1.

\subsection{Chemical cleaning of fouled membrane modules}

During the chemical cleaning process, the fouled membrane module was gently rinsed with pure water and then transferred into a dark brown glass bottle containing $\mathrm{NaClO}$ solution and incubated under shaking. At the end of the cleaning process, residual chlorine of the system was analyzed. Then, the solution was immediately quenched with excessive sodium thiosulphate and subjected to DBP analysis. To investigate the factors influencing DBP formation during membrane cleaning, the fouled membrane was treated with $\mathrm{NaClO}$ solution with the concentration ranging from 500 to $3000 \mathrm{mg} / \mathrm{L}$ (measured as $\mathrm{Cl}_{2}$ ) and $\mathrm{pH}$ ranging from 3 to 12 at various temperature range $\left(15^{\circ} \mathrm{C}-35^{\circ} \mathrm{C}\right)$. Since this study simulated the offline recovery cleaning process of MBRs, high levels of chlorine $(500-3000 \mathrm{mg} / \mathrm{L})$ that predominantly adopted in practical MBRs (Wang et al., 2014) were used. Most of HANs and HKs were below the detection limit at $\mathrm{pH}$ 12.0. Therefore, to clearly show the transformation between DBP species over time, effects of reaction time $(0.5-48 \mathrm{~h})$ on DBP formation conducted at $\mathrm{pH}$ of 3.0 were detailed investigated. The results obtained without $\mathrm{pH}$ adjusting ( $\mathrm{pH}$ of 12) were shown in the Supporting Information.

The residual chlorine was analyzed with a total and free chlorine meter (HI93711, HANNA, Italy). DBPs including THMs [trichloromethane (TCM), bromodichloromethane (BDCM), dibromochloromethane (DBCM) and tribromethane (TBM)], HAAs [dichloroacetic acid (DCAA), monochloroacetic acid (MCAA), and trichloroacetic acid (TCAA)], HANs [trichloroactetonitrile (TCAN), DCAN, bromochloroacetonitrile (BCAN) and dibromoacetonitrile (DBAN)], HKs [1,1,1-trichloropropanone (1,1,1-TCP) and 1,1-dichlro-2-propanone (1,1-DCP)], and trichloronitromethane (TCNM) were analyzed. Detailed determination methods can be found in our previous study (Wang et al., 2020a).

\subsection{EPS Extraction}

The biofilm EPS on the membrane surface was extracted according to the modified heat extraction method (PellicerNàcher et al., 2013). Detailed procedures are present in the Supporting Information Text SI-2. After the heat and centrifugation treatment, the biofilm construction was destroyed, and almost all the EPS and cells were removed from the membrane surface. Membranes after EPS extraction was named as $\mathrm{M}_{1}$. The supernatant was filtrated by a $0.22 \mu \mathrm{m}$ membrane filter. The permeate was EPS solution. The detached cells were primary present in the sediment in the tube $\left(\mathrm{S}_{1}\right)$, and other planktonic cells were retained on the $0.22 \mu \mathrm{m}$ membrane surface $\left(\mathrm{M}_{2}\right)$. After EPS extraction, cells from biofilm were present in $\mathrm{M}_{1}, \mathrm{M}_{0}$ and $\mathrm{M}_{2}$. The mixture of $\mathrm{M}_{1}, \mathrm{M}_{2}$ and $\mathrm{S}_{1}$ were named as EPS- module. To qualitatively study the contribution of EPS on the formation of DBPs in membrane cleaning process, a fouled membrane modules without any treatment and EPS- module were separately treated by immersing in $\mathrm{NaClO}$ solutions (chlorine dose of $1000 \mathrm{mg} / \mathrm{L}$, initial $\mathrm{pH}$ of 12).

\subsection{Multiple fluorescence labeling and CLSM analysis}

The evolution of the composition and structure of biofouling layers on the membrane surface during cleaning process were characterized with LSM900 CLSM (Zeiss, Germany) using a multiple staining method. Total cells, proteins, $\alpha$ - and $\beta$-D-glucopyranose polysaccharides were labeled with SYTO 63, Fluorescein isothiocyanate (FITC), Concanavalin A (ConA), and Calcofluor white (CW), respectively, which were purchased from Thermo Fisher, USA. The detailed procedure of staining can be found in a previous study (Yuan et al., 2015). The excitation laser wavelengths used for SYTO 63, FITC and Con A and CW were $633,488,543$, and $400 \mathrm{~nm}$, respectively.

\section{Results and discussions}

\subsection{Effects of reaction time on the formation of DBPs}

During the cleaning of fouled membranes with $\mathrm{NaClO}$ solution of which chlorine dosage was $1000 \mathrm{mg} / \mathrm{L}$ and $\mathrm{pH}$ and 3.0, the formation of DBPs at different times was investigated and present in Fig. 1.

Besides DBAN, all species were quantifiable among the 14 DBPs of interest. HAAs were the most abundant species, among which TCAA was dominant, composing over $80 \%$ of total DBPs determined in this study. TCM was at the level of hundreds of micrograms per liter. Among the HANs, DCAN was the primary species composing more than $95 \%$ of the total HANs. Total THMs and HAAs accumulated as reaction time prolonged (Figs. 1A and 1D), attributed to the significant and sustained increase of TCM and MCAA, DCAA and TCAA formation, respectively. Over $80 \%$ of the THMs formed in the first $24 \mathrm{~h}$. The yield of TBM gained maximum within the initial $0.5 \mathrm{~h}$ and decreased with prolonged time. The formation of DBCM and BDCM reached highest within the initial $3 \mathrm{~h}$ and decreased thereafter. It indicated that the brominated species was transformed into TCM with reaction time 

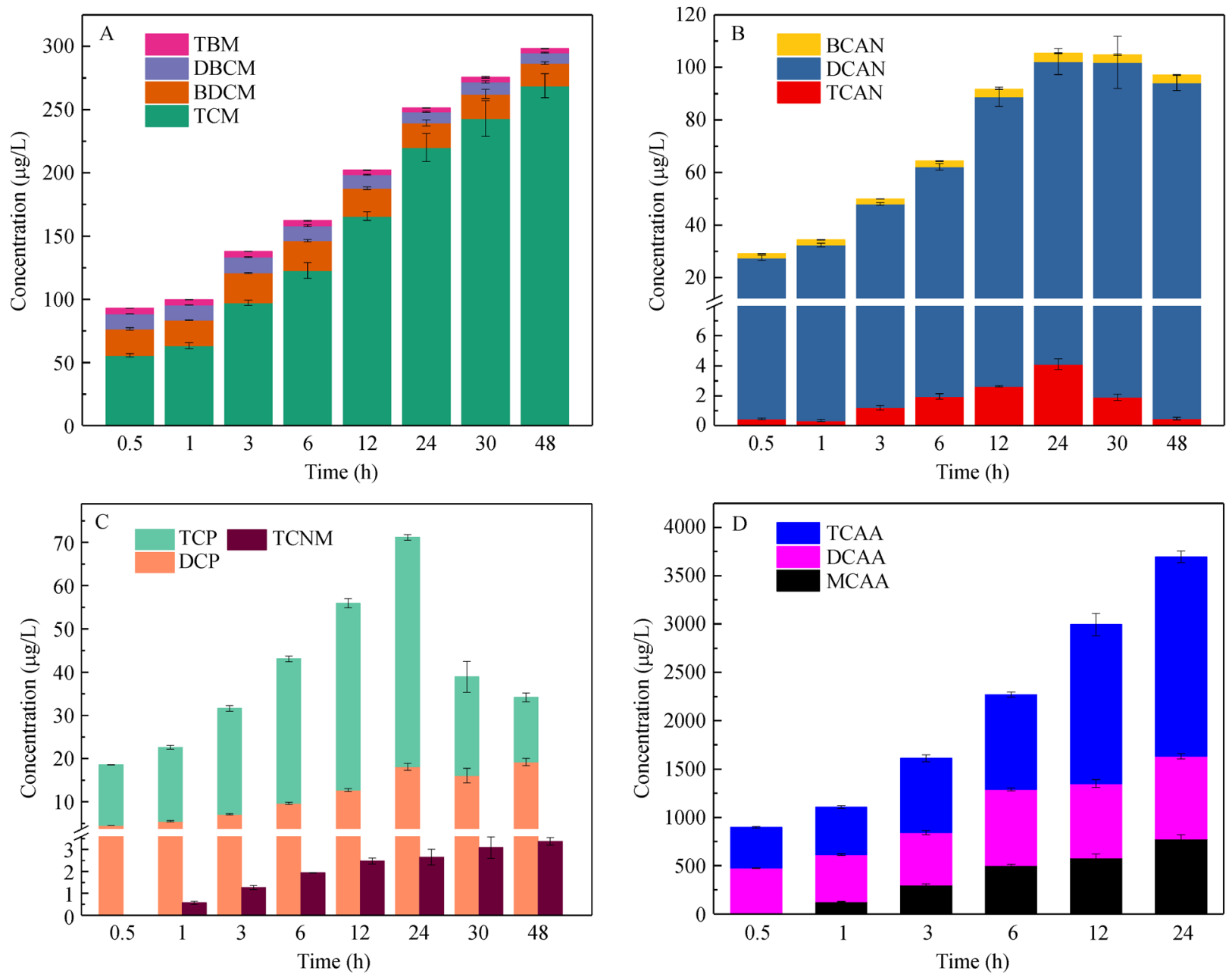

Fig. 1 Effects of reaction time on the formation of (A) THMs, (B) HANs, (C) HKs and TCNM, and (D) HAAs during NaClO cleaning of fouled membranes from an MBR. Reaction conditions: Chlorine dosage was $1000 \mathrm{mg} / \mathrm{L}$; Temperature was $25 \pm 0.1^{\circ} \mathrm{C}$; Initial $\mathrm{pH}$ was 3.0 .

prolonging. As present in Figs. 1B and 1C, yields of total HANs and HKs increased to the maximum within $24 \mathrm{~h}$ and then decreased as reaction time prolonged, which was similar to a previous study (Fang et al., 2010). With reaction time prolonging, the concentration of TCNM increased, which was lower than $3.0 \mu \mathrm{g} / \mathrm{L}$. However, at a strong alkaline condition ( $\mathrm{pH} 12)$, the concentration and formation rate of TCM was increased significantly (Supporting Information Fig. SI-2). More than 90\% of the TCM generated within $12 \mathrm{~h}$. The strong alkaline condition shifted the dominant species from TCAA to DCAA although it had slight influences on the yield of total HAAs.

The formation and decomposition of DBPs determined their time-dependent yield trends. As listed in Supporting Information Table SI-2, THMs and HAAs are much more stable than HANs and HKs (Chen, 2011). TCM and TCAA are usually considered to be the end product of most DBPs due to its high stability in the presence of chlorine. It has been proved that HKs and HANs in the aqueous environment tend to hydrolyze to TCM and TCAA (Fang et al., 2010). Also, the presence of excessive free chlorine residual (more than $100 \mathrm{mg} / \mathrm{L}$ ) accelerated the hydrolysis of HANs (Yang et al., 2007), which enhanced the production of TCM and TCAA. Therefore, the formation of TCM was positively correlated with the reaction time in this study (Fang et al., 2010). Bromide ions present in the $\mathrm{NaClO}$ solution were rapidly oxidized to $\mathrm{HBrO}$, which is a stronger substituting reagent compared with $\mathrm{HClO}$ (Zhu and Zhang, 2016). Therefore, the production of brominated species increased significantly at the beginning of the $\mathrm{NaClO}$ cleaning process. However, compared with TCM, the brominated THMs were less stable (Supporting Information Table SI-2), which might be transformed into TCM through further chlorine substitution with the presence of excessive residual chlorine. 


\subsection{Effects of chlorine dosage on the formation of DBPs}

The formation of a variety DBPs during the cleaning of fouled membranes by $\mathrm{NaClO}$ solutions at various concentrations with the initial $\mathrm{pH}$ of 7.0 was evaluated and is shown in Fig. 2A.

Despite the various chlorine dosage, HAAs were always the most abundant species among the DBPs determined in this study. With the increase of chlorine dosage from 500 to $3000 \mathrm{mg} / \mathrm{L}$, the concentrations of TCAA, DCAA, MCAA, and TCM were increased monotonically. In particular, the concentration of TCAA was increased by a factor of 1.6. Since TCM and TCAA are the end products of most unstable DBPs, their formation is usually positively proportional to chlorine dosage. However, at a strong alkaline condition ( $\mathrm{pH} 12)$, the yield of TCM increased slightly as the chlorine dosage increased from 500 to 3000 $\mathrm{mg} / \mathrm{L}$ (Supporting Information Fig. SI-3). In addition, the production of the other three THMs, BDCM, DBCM, and TBM was increased and then decreased as the chlorine dosage increased from 500 to $3000 \mathrm{mg} / \mathrm{L}$ at acidic $\mathrm{pH}$. This can be explained by that a higher chlorine dosage resulted in a higher $\mathrm{Cl} / \mathrm{Br}$ molar ratio, which enhanced the chlorine substitution while inhibited the bromine substitution (Ma et al., 2015).

In all samples, DCAN was the primary species among HANs investigated in this study. BCAN composed less than $10 \%$ of the HANs in all samples. The concentrations of TCAN and DBAN were too low to quantify. The yield of DCAN was decreased by $88 \%$ as the chlorine dose rose from 1000 to $3000 \mathrm{mg} / \mathrm{L}$. The increase of chlorine dosage resulted in a high level of free chlorine residual in the cleaning solution (higher than $100 \mathrm{mg} / \mathrm{L}$ ), which enhanced the decomposition of HANs to generate more end products, such as TCM and DCAA (Chu et al., 2012). As shown in Supporting Information Table SI-2, TCAN has a much higher hydrolysis rate constant $\left(6.26 \times 10^{-2} \mathrm{~h}^{-1}\right)$ than DCAN (Chen, 2011; Yu and Reckhow, 2015). Therefore, the absence of TCAN in this study might be due to the rapid hydrolysis in the presence of free chlorine. The increase of chlorine dosage resulted in the disappearance of TCP, accompanied by the monotonical increase of DCP, which can also be explained by the high hydrolysis rate constant of TCP (0.182 h-1)(Chen, 2011).

In addition, the rate of flux recovery was positively related to chlorine dosage. About $92 \%$ of the membrane flux was restored as chlorine dosage increased from 500 to 3000 mg/L (Supporting Information Fig. SI-1).

3.3 Effects of initial $\mathrm{pH}$ of cleaning solutions on the formation of DBPs

The yield of DBPs during the cleaning of fouled membranes by $\mathrm{NaClO}$ solutions can also be affected by the initial pH, shown in Fig. 2B. The data show that the concentrations of HAAs were most significantly affected.
The concentration of DCAA increased with rh rising of the initial reaction $\mathrm{pH}$, while the formation of TCAA decreased significantly under the acidic condition. Meanwhile, the formation of TCM was significantly promoted under the alkaline condition. For instance, the yield of TCM was increased by over 14 times when the initial $\mathrm{pH}$ of the cleaning solution was increased from 3.0 to 12.0. In specific, at $\mathrm{pH} 12.0$, the concentration of TCM was as high as $1825.91 \mu \mathrm{g} / \mathrm{L}$. Meanwhile, the production of TBM was also enhanced by $64 \%$, although the formation of BDCM and $\mathrm{DBCM}$ was inhibited. This is consistent with the results from the chlorination of drinking water (Chowdhury et al., 2010). The initial pH of cleaning solutions had great influences on the speciation of HANs and HKs, which are unstable in an aqueous environment. The concentration of TCAN, DCAN, BCAN, TCP, and DCP decreased sharply with the increase of the initial $\mathrm{pH}$. At $\mathrm{pH}$ 12.0 , all these species cannot be quantified because their concentrations were below the detection limit. On the other hand, the concentration of TCNM decreased initially when the $\mathrm{pH}$ changed from 3 to 7 . But it was increased to a higher level when the $\mathrm{pH}$ increased from 7 to 12 . Similar findings were observed in the chlorination of primary amines (Joo and Mitch, 2007). Meanwhile, the flux recovery was accelerated at strong acid or alkaline conditions, which reached $82 \%$ and $85 \%$, respectively (Supporting Information Fig. SI-1).

The effect of $\mathrm{pH}$ on the formation of DBPs during chlorine cleaning of fouled MBR membranes are complex. A higher $\mathrm{pH}$ offered a faster reaction of chlorine consumption by precursors (Ma et al., 2015). Therefore, the formation of some stable DBPs, such as TCM and DCAA, was promoted at a higher $\mathrm{pH}$. However, unstable DBPs, including TCAN, DCAN, BCAN, TCP, and DCP, underwent base-catalyzed hydrolysis decomposition at alkaline conditions, which shifted the products to TCM. Overall, it contributed to the reduction of HANs and HKs concentrations at a higher $\mathrm{pH}$ accompanied by the continuous increase of TCM. Furthermore, R-CO-CX $\mathrm{CX}_{3}$, considered as the precursor of HAAs, tends to transform into TCAA under the acidic conditions when $\mathrm{R}$ is the oxidized functional group. However, at alkaline conditions, the effective base-catalyzed hydrolyzation of R-CO$\mathrm{CX}_{3}$ enhanced the formation of TCM and DCAA. In addition, $\mathrm{pH}$ has great influences on the distribution of active chlorine species. Based on the pKa value of hypochlorous acid $\left(7.53\right.$ at $\left.25^{\circ} \mathrm{C}\right)$, the distirbution of $\mathrm{HClO}$ and $\mathrm{ClO}^{-}$was theoretically calculated and shown in Supporting Information Fig. SI-4. The undecomposed hypochlorous acid $(\mathrm{HClO})$ is the major aqueous chlorine species at a low $\mathrm{pH}$ condition. About $100 \%$ of active chlorine is $\mathrm{HClO}$ when $\mathrm{pH}<4.3$. With the $\mathrm{pH}$ value rising, $\mathrm{HClO}$ gradually deprotonated into hypochlorite ions $\left(\mathrm{ClO}^{-}\right)$. The concentrations of $\mathrm{HClO}$ and $\mathrm{ClO}^{-}$are nearly equal at $\mathrm{pH}=7.54$. Only $\mathrm{ClO}^{-}$is present when $\mathrm{pH}>10.0$. Although $\mathrm{HClO}$ is more efficient to penetrate cells for 

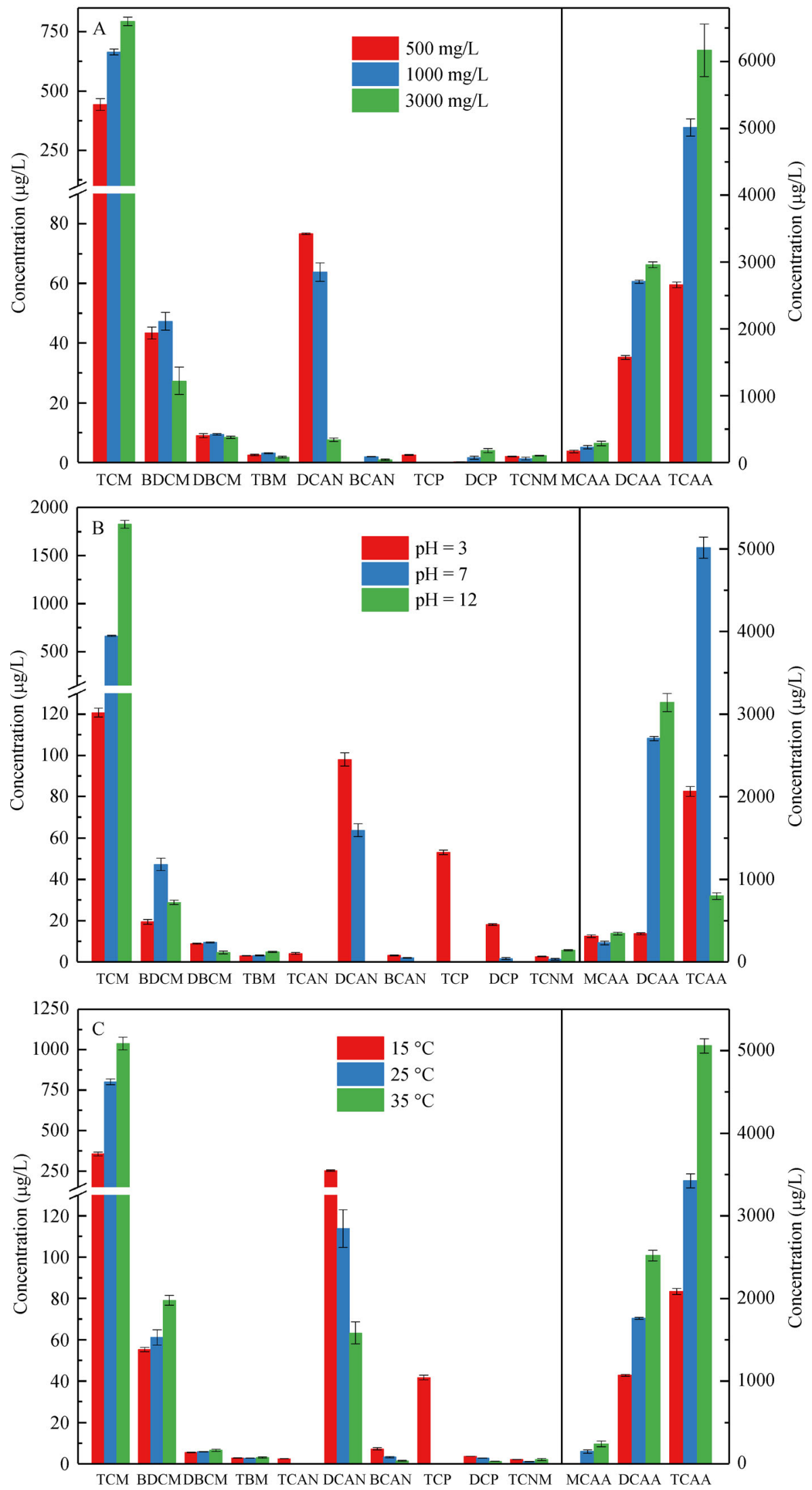

Fig. 2 Effects of (A) chlorine dosage, (B) initial $\mathrm{pH}$, and (C) temperature on the formation of DBPs during NaClO cleaning of fouled membranes from an MBR. The data were obtained after $24 \mathrm{~h}$ of cleaning. The temperature was $25 \pm 0.1^{\circ} \mathrm{C}$ and the initial $\mathrm{pH}$ was $7.0 \pm 0.1$ unless explicitly stated. 
sterilization of microorganisms (Chu et al., 2009), $\mathrm{ClO}^{-}$ exhibits stronger oxidation reactivity (Xie et al., 2015). It has been confirmed that various processes including substitution, addition, oxidation, and hydrolysis were involved in the production of DBPs. Reactive chlorine is primarily involved in oxidation reactions (Luong et al., 1982). Therefore, the alkaline condition promots the production of TCM due to the formation of $\mathrm{ClO}^{-}$. Some chlorination reactions involved in the formation of DBPs can also be accelerated under the alkaline conditions. These include the benzene ring-opening and $\mathrm{C}=\mathrm{O}$ oxidation, which increased the formation of TCM. Additionally, an alkaline condition could improve the hydrophilicity of biofilm on the membrane surface, which enhanced the detachment of biofilm (Alasonati and Slaveykova, 2012). The aqueous biofilm is easier to be penetrated and attacked by active chlorine (Berry et al., 2010), which is in favor of the production of DBPs.

\subsection{Effects of temperature on the formation of DBPs}

The formation and speciation of DBPs after $24 \mathrm{~h}$ cleaning of fouled membranes with $\mathrm{NaClO}$ solution (chlorine dose of $1000 \mathrm{mg} / \mathrm{L}$, initial $\mathrm{pH}$ of 7.0) at various temperatures were illustrated in Fig. 2C. The increase in temperature increased the formation of THMs and HAAs but reduced the concentrations of HANs and HKs. As the temperature was elevated from 15 to $35^{\circ} \mathrm{C}$, the yield of total THMs and HAAs increased by 1.68 times and 2.48 times, respectively. The concentration of TCM formed at $35^{\circ} \mathrm{C}$ was nearly 3 times that formed at $15^{\circ} \mathrm{C}$. On the other hand, the concentrations of DCAN and BCAN decreased by over $75 \%$ when the temperature rose from 15 to $35^{\circ} \mathrm{C}$. The yield of DCP at $35^{\circ} \mathrm{C}$ was less than $35 \%$ of that at $15^{\circ} \mathrm{C}$. At $25^{\circ} \mathrm{C}$ or higher, the concentrations of TCAN and TCP were below the detection limit.

Generally, both the formation and decomposition rates of DBPs could be accelerated with increasing temperature (Yang et al., 2007). For those relative stable DBPs such as THMs and HAAs, the temperature is positively correlated to the rate of production. However, high temperatures increase the decomposition rates of the unstable DBPs, such as HANs and HKs, which are considered as the intermediate species during the formation of THMs (Nikolaou et al., 2000).

3.5 The potential toxicity of DBPs formed at various conditions

Since the cytotoxicity and genotoxicity varied among different DBP species, it is not accurate to evaluate the toxic risks only by examining their concentrations. Plewa and colleagues calculated the combined toxicity value (CTV) of individual DBP species based on its cytotoxicity and genotoxicity to compare the toxicity of different DBPs (Plewa and Wagner, 2015). They found that HANs exhibited extremely higher CTV than THMs (Supporting Information Table SI-3). Here, the toxic risk value (TRV) was applied to evaluate the toxic risks of DBP species formed during the cleaning of the fouled membranes. The TRV of individual DBP species was obtained by multiplying the $C T V_{x}$ with its concentration $C_{x}$ determined in this study (Chu et al., 2016). The total toxicity caused by all DBPs detected in the sample was calculated by summing the TRVs of DBPs, named as the integrated toxic risk value (ITRV). Based on the available data, ITRVs of HAAs, THMs, HANs, and TCNM were calculated, shown in Fig. 3.

From Fig. 3, the total toxic risks of DBPs formed during membrane cleaning are dominated by the HAAs due to their high concentration. The variation of total toxic risks follows the change of ITRV values of HAAs. On the other hand, although the yield of HANs was much lower, they also contributed significantly to the total toxic risks of all DBPs due to their high CTVs. Remarkably, at the low temperature $\left(15^{\circ} \mathrm{C}\right)$ HANs contributed to the total toxic risk more than the HAAs (Fig. $3 \mathrm{D}$ ). Since the CTVs or the formation potentials were low, the contributions from THMs and TCNM to the total toxic risks were insignificant. The toxic risk analysis in Fig. 3 indicates that the cleaning of MBR membranes with a low concentration of $\mathrm{NaClO}$ solution at a low temperature with short reaction time at a non-neutral $\mathrm{pH}$ condition could reduce the toxic risk caused by the formation of DBPs.

In our study, HAAs had significantly higher concentrations than the THMs (more than 10 times). This is contradictory to the results from the sewage and surface water where the yields of THMs and HAAs were close (Sirivedhin and Gray, 2005). However, our observation is consistent with the study about the cleaning of the humic acid-fouled UF membrane (Wang et al., 2018). Thus, the formation of DBPs in membrane cleaning might be unique due to the different precursors involved. Due to their high toxicity and non-volatility, the formation of HAAs during membrane cleaning in MBR must be controlled.

\subsection{Roles of EPS in the formation of DBPs during membrane cleaning}

The EPS extraction, which reduced the content of EPS in the fouled layer, destroyed the structure of the biofilm to a certain extent. Thus the EPS- module lost some of the EPS contributions during the formation of DBPs. The timedependent formation of THMs in the two samples is shown in Fig. 4. It is apparent that the generation of total THMs from the EPS- module was faster than the control module in the initial $12 \mathrm{~h}$. The THM formation rate within the initial $6 \mathrm{~h}$ was increased by $18 \%$ after EPS extraction Nevertheless, the final THM yield after $48 \mathrm{~h}$ reaction in the EPS- module was much lower than the control module. The results implied that EPS had great impacts on the formation rate of THMs. 

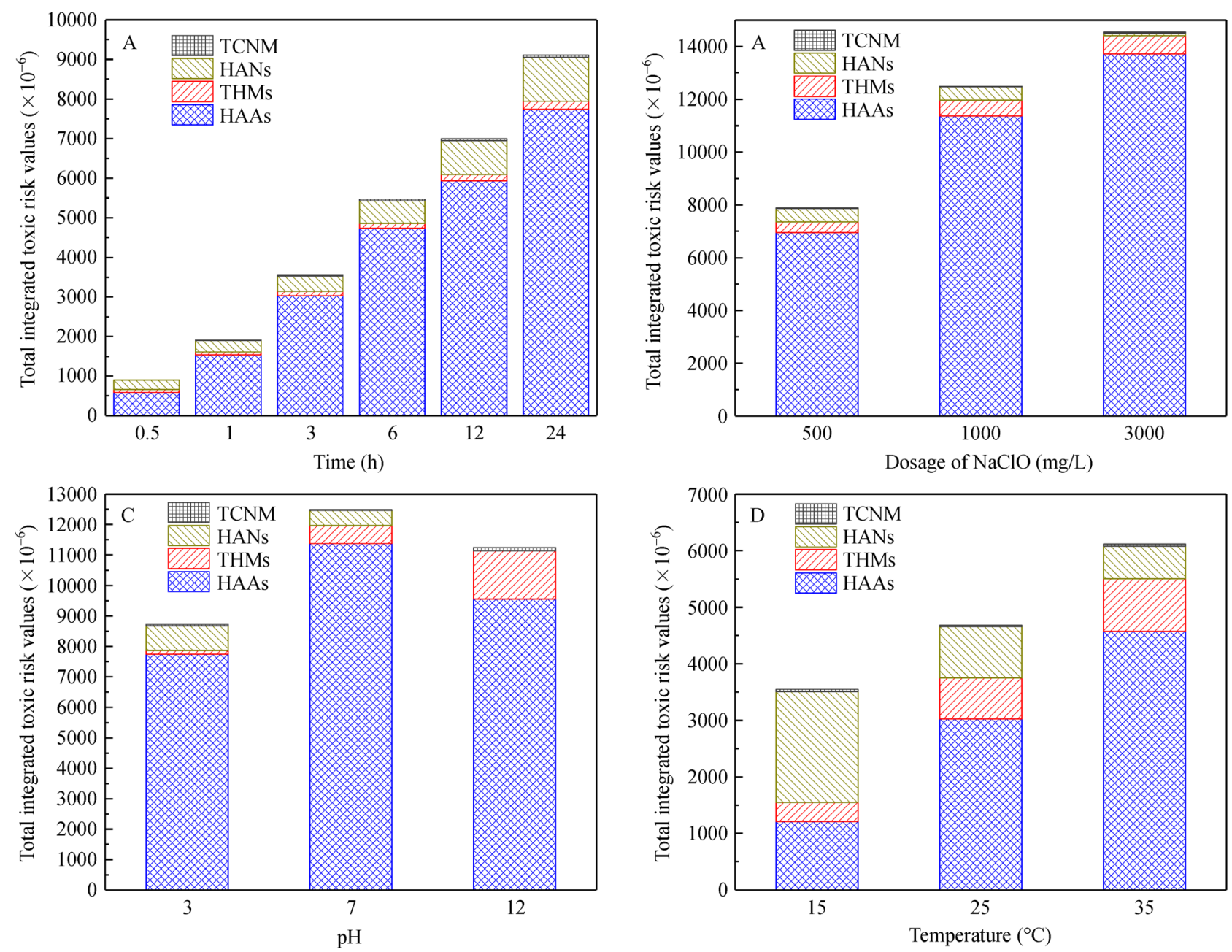

Fig. 3 Effects of reaction time (A), chlorine dosage (B), initial $\mathrm{pH}(\mathrm{C})$, and temperature (D) on the integrated toxic risk values of DBPs formed during cleaning of MBR membranes with $\mathrm{NaClO}$ solution. Reaction conditions (A): Chlorine dosage was $1000 \mathrm{mg} / \mathrm{L}$; Temperature was $25 \pm 0.1^{\circ} \mathrm{C}$; Initial $\mathrm{pH}$ was 3.0; (B): The data were obtained after $24 \mathrm{~h}$ of cleaning; The temperature was $25 \pm 0.1^{\circ} \mathrm{C}$ and the initial $\mathrm{pH}$ was $7.0 \pm 0.1$. (C): The data were obtained after $24 \mathrm{~h}$ of cleaning; Chlorine dosage was $1000 \mathrm{mg} / \mathrm{L}$; The temperature was $25 \pm 0.1^{\circ} \mathrm{C}$. (D): The data were obtained after $2 \mathrm{~h}$ of cleaning; Chlorine dosage was $1000 \mathrm{mg} / \mathrm{L}$ and the initial $\mathrm{pH}$ was $7.0 \pm 0.1$.

Besides substitution, addition, oxidation, and hydrolysis reactions in the chlorination process occurring in homogeneous aqueous solution, the penetration of chlorine into the biofilm and the detachment of biofilm clusters were also important for the formation of DBPs during chlorine cleaning of fouled membranes. It has been proven that gellike EPS can protect the cells inside from chlorine by limiting the transport of this disinfectant into the biofilm (Gordon et al., 1988). Also, EPS could reduce the size of the void between adjacent cells, decreasing the specific surface area, which therefore slowed down the diffusion of the disinfectant into the biofilm (Xue et al., 2012). Therefore, the lack of EPS made the biofilm much easier to be penetrated and oxidized by the active chlorine, which accelerated the formation of THMs. In addition, the biofilm detachment was also promoted due to the lack of protection of EPS, which enhanced its direct contact and subsequently reacted with chlorine, resulting in the accelerated production of THMs.

As the cleaning process proceeded, the biofilm, including cells and EPS, on the membrane surface was detached gradually. The chlorination of suspended or soluble components of detached biofilm in the aqueous phase became the dominant contributor to the formation of THMs. As shown in Supporting Information Fig. SI-5, the extracted EPS also offers high reactivity in producing DBPs. Since the EPS was extracted, the EPS-group had fewer precursors than the control module. Therefore, it was reasonable that the final stable yield of THMs in the EPSmodule was lower than the control module.

The biofilm cultivated on the plate membrane for CLSM imaging was much thinner than that on the hollow fiber membranes in MBRs. Therefore, the evolution of biofilm over time was only investigated qualitatively. Figure 5 presents the evolution of the morphology of the biofouling layer over cleaning time. The images were obtained by 


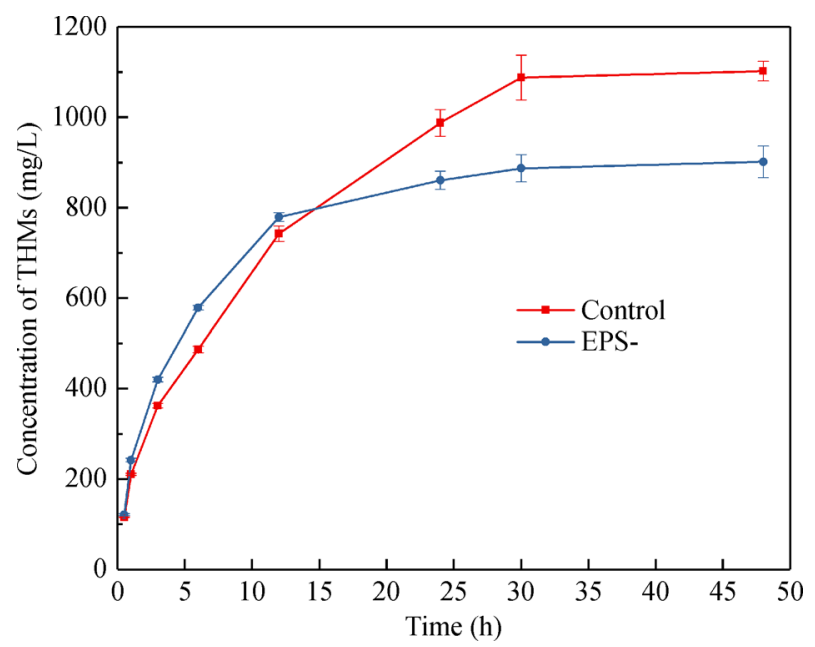

Fig. 4 Effects of biofilm EPS on the formation of THMs during cleaning of fouled membranes with $\mathrm{NaClO}$ solutions. Reaction conditions: Chlorine dosage was $1000 \mathrm{mg} / \mathrm{L}$; Initial $\mathrm{pH}$ was about 12.0. Control represents the fouled membrane module taken from MBR tank without any treatment; EPS- represents the total cells without EPS. merging the CLSM images of proteins, $\alpha$ - and $\beta$-Dglucopyranose polysaccharides, and total cells at the same site.

CLSM side-view images suggest that the fouling layer has a thickness of 12-14 $\mu \mathrm{m}$. The distribution of EPS and cells on the top layer of the biofilm ( $12 \mu \mathrm{m}$ from the surface of substrate, Fig. 5C-a) was much sparser than that in the deeper layer of the biofilm $(2 \mu \mathrm{m}$ from the substrate, Fig. $5 \mathrm{~B}-\mathrm{a})$. After $15 \mathrm{~min}$ of reaction, the top layer of the biofilm had been destroyed significantly, accompanied by the reduction of cells, with a small amount of remaining EPS (Fig. 5C-b). In particular, the reduction of cells was remarkable. However, all substances wereobserved in the CLSM image of deep layer of the biofilm after $15 \mathrm{~min}$ of cleaning treatment (Fig. 5B-b), although the fluorescence intensity from the cells became relatively lower than that from EPS. This is confirmed for the quantitative analysis of the relative fluorescence intensities plotted in Fig. 6. The results suggest that the resistance to chlorine was different for different substances in the biofilm. Cells have poor resistance to chlorine, while proteins and polysaccharides have higher resistance. It also indicates that the efficiency
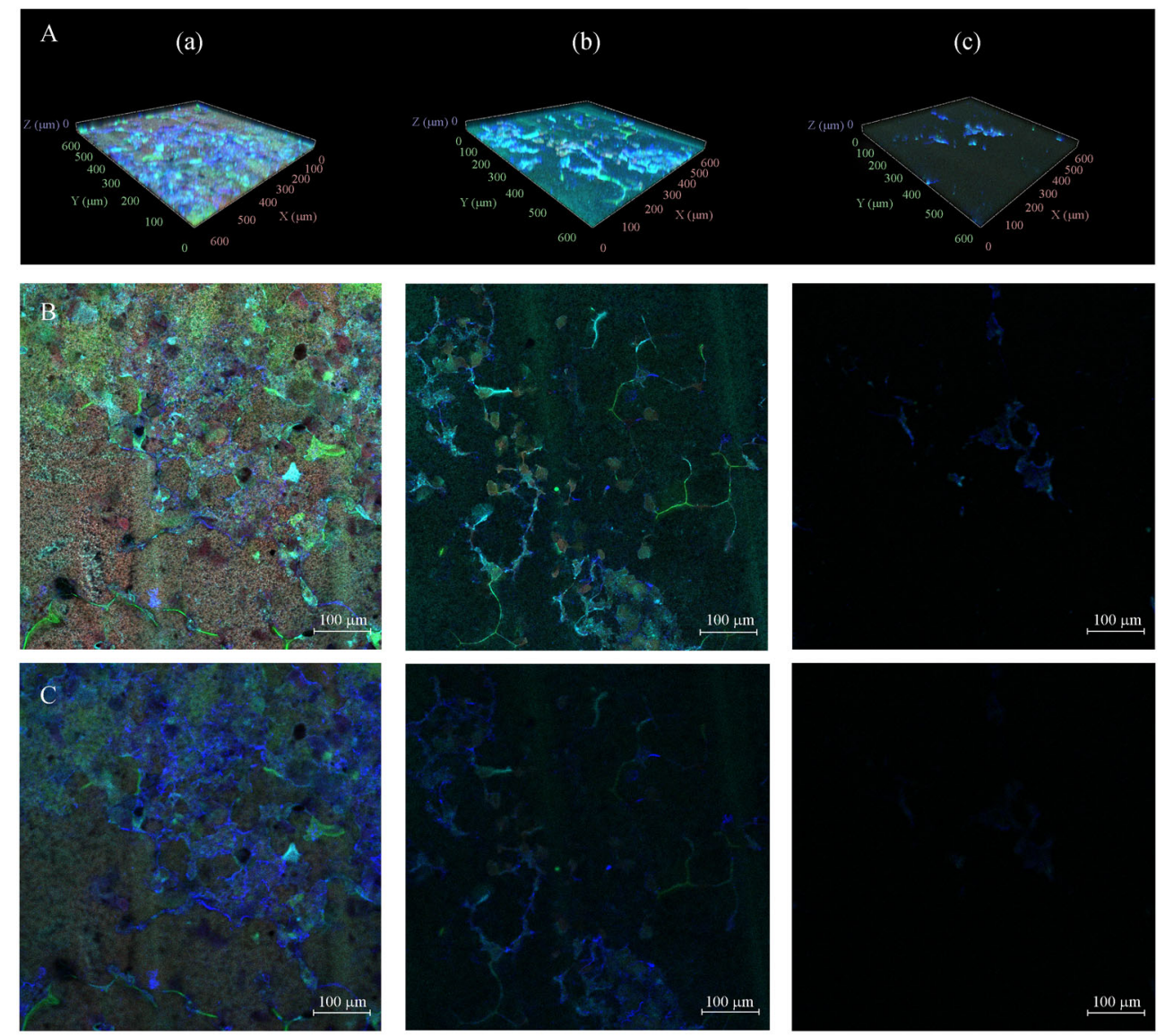

Fig. 5 (A) CLSM z-stack 3-D images of fouling layer including proteins (FITC, green), $\alpha$-D-glucopyranose polysaccharides (Con A, light blue), $\beta$-D-glucopyranose polysaccharides (calcofluor white, blue), and total cells (SYTO 63, red) at (B) $2 \mu \mathrm{m}$ and (C) $12 \mu \mathrm{m}$ above the substrate at different cleaning times, (a) $t=0 \mathrm{~min}$; (b) $t=15 \mathrm{~min}$; (c) $t=60 \mathrm{~min}$; The image area is $638.9 \mu \mathrm{m} \times 638.9 \mu \mathrm{m}$. Cleaning conditions: Chlorine dosage was $1000 \mathrm{mg} / \mathrm{L}$; Initial $\mathrm{pH}$ was $12.0 \pm 0.1$. 


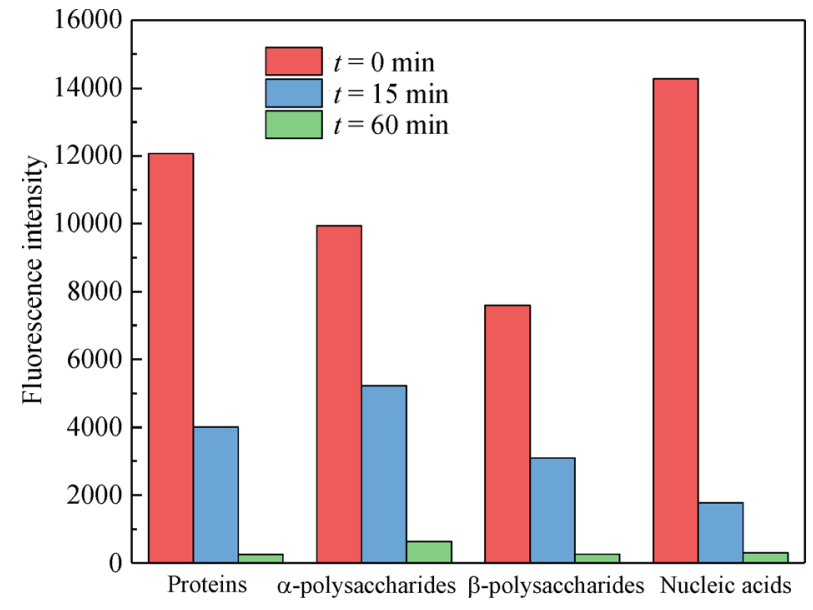

Fig. 6 The integrated fluorescence intensities of proteins, $\alpha$ - and $\beta$-D-glucopyranose polysaccharides, and total cells calculated from CLSM images. The sampling area is $638.9 \mu \mathrm{m} \times 638.9 \mu \mathrm{m} \times$ $10 \mu \mathrm{m}$.

of fouling layer destruction in $\mathrm{NaClO}$ cleaning was regulated by the remaining EPS. After 15 min of cleaning treatment, the membrane permeability was recovered by about 55\% (Supporting Information Fig. SI-1). After $60 \mathrm{~min}$ of cleaning, the membrane permeability was recovered about $85 \%$ and the top layer of the biofilm was almost completely detached (Fig. 5C-c). Only a small amount of polysaccharides were left on the surface, while no cells or proteins were observed. Meanwhile, the inner layer of the biofilm ( $2 \mu \mathrm{m}$ from the substrate) was also significantly destroyed. However, there were clusters of substance remaining contained cells and EPS dominated by $\beta$-polysaccharides. (Fig. 5B-c). Nonetheless, most areas had been completely cleaned after $60 \mathrm{~min}$ of cleaning treatment. This confirmed that the presence of EPS could protect the cells in chlorination, which reduces the effectiveness of disinfection due to the restricted penetration of chlorine through the gel-EPS. As the top layer of the biofilm was removed, the disinfectant gradually penetrated the deeper layer, resulting in the detachment of EPS and cells through chlorination and sterilization.

\section{Conclusions}

HAAs were the most abundant species and the dominant contributor to the toxicity of the DBPs of interest. Increasing the cleaning time, chlorine dosage, reaction temperature, and solution $\mathrm{pH}$ enhanced the formation of TCM and HAAs but reduced HANs and HKs due to the accelerated hydrolysis. The decrease in the cleaning time, chlorine dosage and temperature, or adjust the solution $\mathrm{pH}$ away from natural can effectively reduce the toxic risks caused by the formation of DBPs. A considerable proportion of DBPs formed during membrane cleaning was derived from chlorinated EPS. EPS, particularly polysaccharides, was highly resistant to chlorine. Although the resistance of cells to chlorine in the biofilm was low, the presence of EPS can restrict the penetration of chlorine into the cells, which decelerated the production of DBPs.

Acknowledgements This work was supported by the National Natural Science Foundation of China (Grant No. 51808316) and the Natural Science Foundation of Shandong Province (Grant No. ZR2019BEE015). In addition, we thank Xiaomin Zhao, Haiyan Yu, and Sen Wang from the State Key Laboratory of Microbial Technology for assistance with confocal imaging.

Electronic Supplementary Material Supplementary material is available in the online version of this article at https://doi.org/10.1007/s11783-021$1389-3$ and is accessible for authorized users.

\section{References}

Alasonati E, Slaveykova V I (2012). Effects of extraction methods on the composition and molar mass distributions of exopolymeric substances of the bacterium Sinorhizobium meliloti. Bioresource Technology, 114: 603-609

Berry D, Holder D, Xi C, Raskin L (2010). Comparative transcriptomics of the response of Escherichia coli to the disinfectant monochloramine and to growth conditions inducing monochloramine resistance. Water Research, 44(17): 4924-4931

Cai W, Han J, Zhang X, Liu Y (2020). Formation mechanisms of emerging organic contaminants during on-line membrane cleaning with $\mathrm{NaOCl}$ in MBR. Journal of Hazardous Materials, 386: 121966

Cai W, Liu J, Zhu X, Zhang X, Liu Y (2017). Fate of dissolved organic matter and byproducts generated from on-line chemical cleaning with sodium hypochlorite in MBR. Chemical Engineering Journal, 323: 233-242

Chen B (2011). Hydrolytic stabilities of halogenated disinfection byproducts: Review and rate constant quantitative structure-property relationship analysis. Environmental Engineering Science, 28(6): 385-394

Chowdhury S, Champagne P, James Mclellan P (2010). Investigating effects of bromide ions on trihalomethanes and developing model for predicting bromodichloromethane in drinking water. Water Research, 44(7): 2349-2359

Chu W, Gao N, Krasner S W, Templeton M R, Yin D (2012). Formation of halogenated C-, N-DBPs from chlor(am)ination and UV irradiation of tyrosine in drinking water. Environmental Pollution, 161: 8-14

Chu W, Li X, Bond T, Gao N, Bin X, Wang Q, Ding S (2016). Copper increases reductive dehalogenation of haloacetamides by zero-valent iron in drinking water: Reduction efficiency and integrated toxicity risk. Water Research, 107: 141-150

Chu W H, Gao N Y, Deng Y, Dong B Z (2009). Formation of chloroform during chlorination of alanine in drinking water. Chemosphere, 77(10): 1346-1351

Ding A, Zhao Y, Ngo H H, Bai L, Li G, Liang H, Ren N, Nan J (2020a). Metabolic uncoupler, 3,3', $4^{\prime}, 5$-tetrachlorosalicylanilide addition for sludge reduction and fouling control in a gravity-driven membrane bioreactor. Frontiers of Environmental Science \& Engineering, 14(6): 
96

Ding A, Zhao Y, Yan Z, Bai L, Yang H, Liang H, Li G, Ren N (2020b). Co-application of energy uncoupling and ultrafiltration in sludge treatment: Evaluations of sludge reduction, supernatant recovery and membrane fouling control. Frontiers of Environmental Science \& Engineering, 14(4): 59

Fang J, Ma J, Yang X, Shang C (2010). Formation of carbonaceous and nitrogenous disinfection by-products from the chlorination of Microcystis aeruginosa. Water Research, 44(6): 1934-1940

Garrido-Baserba M, Asvapathanagul P, Mccarthy G W, Gocke T E, Olson B H, Park H D, Al-Omari A, Murthy S, Bott C B, Wett B, Smeraldi J D, Shaw A R, Rosso D (2016). Linking biofilm growth to fouling and aeration performance of fine-pore diffuser in activated sludge. Water Research, 90: 317-328

Gordon C A, Hodges N A, Marriott C (1988). Antibiotic interaction and diffusion through alginate and exopolysaccharide of cystic fibrosisderived Pseudomonas aeruginosa. Journal of Antimicrobial Chemotherapy, 22(5): 667-674

Guo X, Li C, Li C, Wei T, Tong L, Shao H, Zhou Q, Wang L, Liao Y (2019). G-CNTs/PVDF mixed matrix membranes with improved antifouling properties and filtration performance. Frontiers of Environmental Science \& Engineering, 13(6): 81

Joo S H, Mitch W A (2007). Nitrile, aldehyde, and halonitroalkane formation during chlorination/chloramination of primary amines. Environmental Science \& Technology, 41(4): 1288-1296

Luong T V, Peters C J, Perry R (1982). Influence of bromide and ammonia upon the formation of trihalomethanes under watertreatment conditions. Environmental Science \& Technology, 16(8): 473-479

Lv L, Jiang T, Zhang S, Yu X (2014). Exposure to mutagenic disinfection byproducts leads to increase of antibiotic resistance in Pseudomonas aeruginosa. Environmental Science \& Technology, 48 (14): 8188-8195

Ma D, Gao B, Wang Y, Yue Q, Li Q (2015). Factors affecting trihalomethane formation and speciation during chlorination of reclaimed water. Water Science and Technology, 72(4): 616-622

Nikolaou A D, Golfinopoulos S K, Kostopoulou M N, Lekkas T D (2000). Decomposition of dihaloacetonitriles in water solutions and fortified drinking water samples. Chemosphere, 41(8): 1149-1154

Pellicer-Nàcher C, Domingo-Félez C, Mutlu A G, Smets B F (2013). Critical assessment of extracellular polymeric substances extraction methods from mixed culture biomass. Water Research, 47(15): 5564 5574

Plewa, M J, Wagner E D. Charting a new path to resolve the adverse health effects of DBPs (2015). In: Karanfil T, Mitch B, Westerhoff B, Xie Y F. Recent advances in disinfection by-products. Washington, DC: American Chemical Society, 1190: 3-23

Sirivedhin T, Gray K A (2005). 2. Comparison of the disinfection byproduct formation potentials between a wastewater effluent and surface waters. Water Research, 39(6): 1025-1036

Sun H, Liu H, Han J, Zhang X, Cheng F, Liu Y (2018). Chemical cleaning-associated generation of dissolved organic matter and halogenated byproducts in ceramic MBR: Ozone versus hypochlorite. Water Research, 140: 243-250

Wang H, Shi W, Ma D, Shang Y, Wang Y, Gao B (2020a). Formation of DBPs during chlorination of antibiotics and control with permanganate/bisulfite pretreatment. Chemical Engineering Journal, 392: 123701

Wang S, Chew J W, Liu Y (2020b). An environmentally sustainable approach for online chemical cleaning of MBR with activated peroxymonosulfate. Journal of Membrane Science, 600: 117872

Wang Z, Ding J, Xie P, Chen Y, Wan Y, Wang S (2018). Formation of halogenated by-products during chemical cleaning of humic acidfouled UF membrane by sodium hypochlorite solution. Chemical Engineering Journal, 332: 76-84

Wang Z, Ma J, Tang C Y, Kimura K, Wang Q, Han X (2014). Membrane cleaning in membrane bioreactors: A review. Journal of Membrane Science, 468: 276-307

Wu Q, Yan Y, Lu Y, Du Y, Liang Z, Hu H (2020). Identification of important precursors and theoretical toxicity evaluation of byproducts driving cytotoxicity and genotoxicity in chlorination. Frontiers of Environmental Science \& Engineering, 14(2): 25

Xiao K, Xu Y, Liang S, Lei T, Sun J, Wen X, Zhang H, Chen C, Huang $X$ (2014). Engineering application of membrane bioreactor for wastewater treatment in China: Current state and future prospect. Frontiers of Environmental Science \& Engineering, 8(6): 805-819

Xie P, De Lannoy C F, Ma J, Wiesner M R (2015). Chlorination of polyvinyl pyrrolidone-polysulfone membranes: Organic compound release, byproduct formation, and changes in membrane properties. Journal of Membrane Science, 489: 28-35

Xue Z, Sendamangalam V R, Gruden C L, Seo Y (2012). Multiple roles of extracellular polymeric substances on resistance of biofilm and detached clusters. Environmental Science \& Technology, 46(24): 13212-13219

Yang X, Shang C, Westerhoff P (2007). Factors affecting formation of haloacetonitriles, haloketones, chloropicrin and cyanogen halides during chloramination. Water Research, 41(6): 1193-1200

Yu Y, Reckhow D A (2015). Kinetic analysis of haloacetonitrile stability in drinking waters. Environmental Science \& Technology, 49(18): 11028-11036

Yuan B, Wang X, Tang C, Li X, Yu G (2015). In situ observation of the growth of biofouling layer in osmotic membrane bioreactors by multiple fluorescence labeling and confocal laser scanning microscopy. Water Research, 75: 188-200

Zhang X, Liu Y (2020). Potential toxicity and implication of halogenated byproducts generated in MBR online - cleaning with hypochlorite. Journal of Chemical Technology and Biotechnology (Oxford, Oxfordshire), 95(1): 20-26

Zhu X, Zhang X (2016). Modeling the formation of TOCl, TOBr and TOI during chlor(am)ination of drinking water. Water Research, 96: $166-176$ 